\title{
The selfnegadual properties of generalised quadratic Boolean functions
}

\author{
Lars Eirik Danielsen • Matthew G. Parker
}

Received: date / Accepted: date

\begin{abstract}
We define and characterise selfnegadual generalised quadratic Boolean functions by establishing a link, both to the multiplicative order of symmetric binary matrices, and also to the Hermitian self-dual $\mathbb{F}_{4}$-linear codes. This facilitates a novel way to classify Hermitian self-dual $\mathbb{F}_{4}$-linear codes.
\end{abstract}

Keywords Generalised Boolean functions, selfnegadual functions, negabent functions, bent functions, negaHadamard transform, Walsh Hadamard transform, Fourier eigenspectra, selfdual codes, quantum codes

\section{Introduction}

In this paper an $n$-variable generalised quadratic Boolean function refers to a function in $\operatorname{ZRM}(2, n)$ (the quaternary Reed-Muller code). To be explicit, if $f$ : $\mathbb{F}_{2}^{n} \rightarrow \mathbb{Z}_{4}$ is of the form:

$$
f(x)=2\left(\sum_{j<k} a_{j k} x_{j} x_{k}\right)+2\left(\sum_{j} b_{j} x_{j}+c\right)+\sum_{j} a_{j j} x_{j}+d
$$

where $a_{j k}, a_{j j}, b_{j}, c, d \in \mathbb{F}_{2}$, then it is a function in $\operatorname{ZRM}(2, n)$. We consider $f$ and its phase representation, $i^{f}$, where $i^{2}=-1$, and $i^{f}=\left(i^{f(x)}, x \in \mathbb{F}_{2}^{n}\right)$, is interpreted as a column vector of $2^{n}$ elements. When $a_{j j}=d=0, \forall j$, then $f / 2$ is a quadratic Boolean function. We consider a map from $f$ to an $n \times n$ binary symmetric matrix, $A_{f}=\left(a_{i j}\right)$, where the off-diagonal part refers to Boolean quadratic terms, the diagonal part refers to $\mathbb{Z}_{4}$-linear terms, and the binary linear coefficients, $b_{i}$, together with the constant term, $c$, is called the binary affine offset, and is ignored for the map to a matrix. To simplify notation, and without loss of generality, we assume, throughout this paper, that $c=d=0$.

This work was supported by the Norwegian Research Council (NFR).

Danielsen and Parker

The Selmer Center, Department of Informatics, University of Bergen, PB 7800, N-5020 Bergen, Norway,

E-mail: larsed@ii.uib.no, matthew@ii.uib.no 
Recent papers $[4,8,11]$ have classified and constructed Boolean functions that are selfdual, i.e. their phase representations are eigenvectors of the

Walsh-Hadamard transform - such functions are therefore bent. Another direction is to classify and construct Boolean functions that are negabent or bent-negabent (both bent and negabent) $[15,16]$. We show that a function in $\operatorname{ZRM}(2, n)$ can never be both selfdual and negabent. Let a function be called selfnegadual if its phase representation is an eigenvector of the negaHadamard transform - such functions are therefore negabent and it turns out that they are also bent. We show that there are no quadratic selfnegadual Boolean functions, but that there are selfnegadual functions in $\operatorname{ZRM}(2, n)$. So, in this paper, we answer the following question:

Question 1. Which functions in $\operatorname{ZRM}(2, n)$ are selfnegadual?

The main result of this paper is to characterise those functions in $\operatorname{ZRM}(2, n)$ that are selfnegadual.

We came at this problem from an unusual direction. Our initial question was in the context of the study of multiplicative orders of symmetric binary matrices. Let $A$ be such a matrix, and let it have order $p$ if $A^{p}=I$, the identity, and $A^{j} \neq I$, for $1 \leq j<p$. We say that $\operatorname{ord}(A)=p$, where $A$ can only have an order if $A$ has maximum rank. If $f \in \operatorname{ZRM}(2, n)$ is selfdual, then $\operatorname{ord}\left(A_{f}\right)=2$ [4]. If $f$ is negabent then $A_{f}+I$ has maximum rank [15]. However, if $\operatorname{ord}\left(A_{f}\right)=2$ then $A_{f}+I$ cannot have maximum rank. This motivates the question:

Question 2. For which $n \times n$ symmetric binary matrices, $A$, is $\operatorname{ord}(A)$ and $\operatorname{ord}(A+I)$ jointly minimised to 3 ?

An $n \times n$ symmetric binary matrix, $A$, represents an $n$-vertex undirected graph with possible loops. For $\omega$ primitive in $\mathbb{F}_{4}$, the rowspace of matrix $A+w I$ is a Hermitian selfdual $\mathbb{F}_{4}$-additive code of blocklength $n$. The graphical interpretation has been used to aid in classifying all selfdual $\mathbb{F}_{4}$-additive codes up to blocklength 12 [7]. A small subset of these matrices, $A+\omega I$, generate selfdual $\mathbb{F}_{4}$-additive codes that are also selfdual $\mathbb{F}_{4}$-linear of blocklength $n$ - all selfdual $\mathbb{F}_{4}$-linear codes can be represented in this way. So we have the question:

Question 3. For which $n \times n$ symmetric binary matrices, $A$, is the $\mathbb{F}_{4}$-additive code generated by $A+w I$ also $\mathbb{F}_{4}$-linear?

Question 3 has been characterised by Van den Nest [17]. Our contribution is to show that questions 2 and 3 are the same question, and also the same as question 1 to within a binary affine offset.

In section 2 we characterise dualities of functions with respect to WalshHadamard and negaHadamard transforms. In section 3 we look at orders of symmetric binary matrices, show how they relate to dualities of a function, and show that order is preserved by action of the orthogonal group. In section 4 we show how selfduality and selfnegaduality relate to linear selfdual codes over $\mathbb{F}_{2}$ and $\mathbb{F}_{4}$, respectively, and also, by interpreting the symmetric matrix as an undirected graph, show how a modified form of local complementation [2] on the graph preserves selfnegaduality. In section 5 we use graphical interpretation, and orthogonal equivalence, to classify all Hermitian selfdual $\mathbb{F}_{4}$-linear codes up to $n=18$. 


\section{Bentness and selfdualities of functions - definitions}

For two length- $n$ vectors, $u=\left(u_{0}, u_{1}, \ldots, u_{n-1}\right)^{T}$ and $v=\left(v_{0}, v_{1}, \ldots, v_{n-1}\right)^{T}$, let $u \cdot v=\sum_{j=0}^{n-1} u_{j} v_{j}$ be the dot-product of $u$ and $v$, and $u v=\left(u_{0} v_{0}, u_{1} v_{1}, \ldots, u_{n-1} v_{n-1}\right)^{T}$. Let $w(x)$ be the Hamming weight of $x$.

For $f: \mathbb{F}_{2}^{n} \rightarrow \mathbb{Z}_{4}$ in $\operatorname{ZRM}(2, n)$, let $H=\frac{1}{\sqrt{2}}\left(\begin{array}{rr}1 & 1 \\ 1 & -1\end{array}\right)$ be the $2 \times 2$ Hadamard matrix, and $N=\frac{1}{\sqrt{2}}\left(\begin{array}{rr}1 & i \\ 1 & -i\end{array}\right), i^{2}=-1$, be the $2 \times 2$ negaHadamard matrix. Let ' $\otimes$ ' indicate the tensor product of matrices.

We define $f$ to be a bent function in $\operatorname{ZRM}(2, n)$ if

$$
F_{k}=2^{-n / 2} \sum_{j \in \mathbb{F}_{2}^{n}} i^{f(x)+2 j \cdot k},
$$

satisfies $\left|F_{k}\right|=1, \forall k \in \mathbb{F}_{2}^{n}$. Alternatively we can write

$$
F=H^{\otimes n} i^{f} .
$$

In such a case we can define the dual of $f$ by $i^{\hat{f}}=F$, where $\hat{f}$ is also a bent function in $\operatorname{ZRM}(2, n)$.

We define $f$ to be a negabent function in $\operatorname{ZRM}(2, n)$ if

$$
F_{k}=\alpha 2^{-n / 2} \sum_{j \in \mathbb{F}_{2}^{n}} i^{f(x)+2 j \cdot k+w(j)},
$$

satisfies $\left|F_{k}\right|=1, \forall k \in \mathbb{F}_{2}^{n}$, where $\alpha$ is an arbitrary constant that satisfies $|\alpha|=1$. Alternatively we can write

$$
F=\alpha N^{\otimes n} i^{f} .
$$

In such a case we can define the negadual of $f$ by $i^{\tilde{f}}=F$, where $\tilde{f}$ is then a bent function in $\operatorname{ZRM}(2, n)$.

We summarise the bent and negabent properties in Table 1, as well as the dualities that we discuss in this paper, where $s, r, r^{\prime} \in \mathbb{F}_{2}^{n}$. For brevity, except in the case of selfdual and selfnegadual, we do not make explicit the global multiplicative constants or eigenvalues in these expressions, using symbols $\alpha$ and $\alpha^{\prime}$ to indicate arbitrary constants that need only satisfy $|\alpha|=\left|\alpha^{\prime}\right|=1$. In [4] the cases of $\alpha=1$ and $\alpha=-1$ were used to distinguish between selfdual and anti-selfdual, respectively, but we refer, here to the union of these two simply as selfdual. For selfnegadual, observe that $N^{3}=e^{\pi i / 4} I$. For some eigenvalue, $\alpha$, and eigenvector, $v$, of $N^{\otimes n}$, we have $N^{\otimes n} v=\alpha v$. Therefore $\left(N^{\otimes n}\right)^{3} v=\alpha^{3} v=e^{n \pi i / 4} v$. Therefore $\alpha \in$ $\left\{e^{(n+8 k) \pi i / 12}, k=0,1,2\right\}$. Choosing $f$ from $\operatorname{ZRM}(2, n)$ and selfnegadual restricts $\alpha$ to the alphabet $\left\{e^{h i \pi / 4}\right\}$, for some integer, $h$. Theorem 2 and lemma 12 will show that, for such an $f$, then $n$ must be even. It then follows, uniquely, that $\alpha=e^{-n i \pi / 4}$.

If $f$ is bent then its dual, $\hat{f}$, is also bent. But if $f$ is negabent, then its dual, $\tilde{f}$, is only negabent if $f$ is also bent. A selfdual function is bent and a selfnegadual function is bent-negabent, where bentness of the selfnegadual function follows because $H^{\otimes n} i^{f(x)}=e^{n i \pi / 4} H^{\otimes n} N^{\otimes n} i^{f(x)}=e^{n i \pi / 4} i^{f(x)+\sum_{j} x_{j}}$.

Selfdual and selfnegadual are special cases of $P$-dual and $P$-negadual, respectively. If $f-\hat{f}$ or $f-\tilde{f}$ are of the form $2 r \cdot x+c$, for some $r \in \mathbb{F}_{2}^{n}, c \in \mathbb{F}_{4}$, then $f$ is $\mathrm{P}$-dual or $\mathrm{P}$-negadual, respectively. 
Table 1 Spectral dualities

\begin{tabular}{|c|c|}
\hline Property of $f \in \mathrm{ZRM}(2, n)$ & equation satisfied by $f$ \\
\hline bent & $i^{\hat{f}}=H^{\otimes n} i^{f}$ \\
\hline negabent & $i^{\tilde{f}}=\alpha N^{\otimes n} i^{f}$ \\
\hline selfdual & $i^{f}= \pm H^{\otimes n} i^{f}$ \\
\hline selfnegadual & $i^{f}=e^{-n i \pi / 4} N^{\otimes n} i^{f}$ \\
\hline P-dual & $\begin{aligned} i^{f(x)} & =\alpha H^{\otimes n} i^{f(x+s)+2 r \cdot x} \\
& =\alpha^{\prime} H^{\otimes n} i f(x)+2 r^{\prime} \cdot x\end{aligned}$ \\
\hline P-negadual & $\begin{aligned} i^{f(x)} & =\alpha N^{\otimes n} i^{f(x+s)+2 r \cdot x} \\
& =\alpha^{\prime} N^{\otimes n} i^{f(x)+2 r^{\prime} \cdot x}\end{aligned}$ \\
\hline
\end{tabular}

\section{Matrix orders and function dualities}

We wish to characterise the $n \times n$ binary symmetric matrices, $A$, such that both $\operatorname{ord}(A)$ and $\operatorname{ord}(A+I)$ are as small as possible. We call $A$ a $(p, q)$-matrix if $A$ has order $p$ and $A+I$ has order $q$, where $p$ or $q$ equals ' - ' if $A$ or $A+I$, respectively, does not have maximum rank. Trivially, if $\operatorname{ord}(A)=1$, then $A=I$ and $A+I$ cannot have maximum rank, in which case $A$ is a $(1,-)$-matrix. Likewise, if $\operatorname{ord}(A)=2$ then $A+I$ cannot have maximum rank, as $(A+I)^{2}=A^{2}+I=0$, in which case $A$ is a $(2,-)$-matrix. One can trivially obtain $(-, 1)$ and $(-, 2)$ matrices by replacing $A$ by $A+I$ in the above. So our first candidate of interest is for $A$ to be a $(3,3)$-matrix and, indeed, such symmetric binary matrices do exist. After some preliminary lemmas, we present, by considering the conditions on $f \in \operatorname{ZRM}(2, n)$ for selfduality and selfnegaduality, two theorems for $(2,-)$ matrices (theorem 1 ), and $(3,3)$ matrices (theorem 2$)$, respectively.

Let $X=\left(\begin{array}{ll}0 & 1 \\ 1 & 0\end{array}\right)$ and $Z=\left(\begin{array}{rr}1 & 0 \\ 0 & -1\end{array}\right)$. The following lemma is easily verified.

\section{Lemma 1}

$$
\begin{array}{ll}
H X=Z H, & H Z=X H, \\
N X=i Z X N, & N Z=X N .
\end{array}
$$

For $r \in \mathbb{F}_{2}^{n}, U$ a $2 \times 2$ matrix, $U^{0}=I$, the identity, and $U^{1}=U$, let $U_{r}=$ $\bigotimes_{0 \leq j<n} U^{r_{j}}$, be a $2^{n} \times 2^{n}$ matrix constructed using the tensor product.

Lemma 2 For $f$ bent,

$$
H^{\otimes n} i^{f(x+r)}=i^{\hat{f}(x)+2 r \cdot x} .
$$

Proof. By lemma 1 and Table 1,

$$
\begin{aligned}
& H^{\otimes n} i^{f(x+r)}=H^{\otimes n} X_{r} i^{f(x)} \\
& \quad=Z_{r} H^{\otimes n} i^{f(x)}=Z_{r} i^{\hat{f}(x)}=i^{\hat{f}(x)+2 r \cdot x} .
\end{aligned}
$$


Lemma 3 For $f$ negabent,

$$
N^{\otimes n} i^{f(x+r)}=\alpha i^{\tilde{f}(x+r)+2 r \cdot x+w(r)},
$$

and

$$
N^{\otimes n} i^{f(x)+2 r \cdot x}=\alpha i^{\tilde{f}(x+r)} .
$$

Proof. By lemma 1 and Table 1,

$$
\begin{aligned}
& N^{\otimes n} i^{f(x+r)}=N^{\otimes n} X_{r} i^{f(x)} \\
& \quad=i^{w(r)} Z_{r} X_{r} N^{\otimes n} i^{f(x)}=\alpha Z_{r} X_{r} i^{\tilde{f}(x)+w(r)}=\alpha i^{\tilde{f}(x+r)+2 r \cdot x+w(r) .} .
\end{aligned}
$$

For the second part,

$$
N^{\otimes n} i^{f(x)+2 r \cdot x}=N^{\otimes n} Z_{r} i^{f(x)}=X_{r} N^{\otimes n} i^{f(x)}=\alpha X_{r} i^{\tilde{f}(x)}=\alpha i^{\tilde{f}(x+r)} .
$$

Lemma 4 For $f \in Z R M(2, n)$, and for any $r \in \mathbb{F}_{2}^{n}$,

$$
f(x+r)=f(x)+2 A_{f} r \cdot x+f(r)-f(0) .
$$

Proof. From (1), $f(x)=2 q(x)+2 b \cdot x+a \cdot x+f(0)$, where $q$ is a homogeneous quadratic, and $b, a \in \mathbb{F}_{2}^{n}$. Then $2 q(x+r)=2\left(q(x)+A_{q} r \cdot x+q(r)\right), 2 b \cdot(x+r)=$ $2 b \cdot x+2 b \cdot r$, and $a \cdot(x+r)=a \cdot x+2 a r \cdot x+a \cdot r$. Therefore $f(x+r)=2 q(x+r)+2 b \cdot(x+$ $r)+a \cdot(x+r)+f(0)=(2 q(x)+2 b \cdot x+a \cdot x+f(0))+2\left(A_{q} r+a r\right) \cdot x+(2 q(r)+2 b \cdot r+a \cdot r)$. Observe that $A_{f} r=A_{q} r+a r$.

Theorem 1 If $f$ is selfdual or anti-selfdual then $A_{f}$ is a $(2,-)$-matrix, and $f(r)=$ $-f\left(A_{f} r\right)+2 f(0), \forall r \in \mathbb{F}_{2}^{n}$. Conversely, if $A_{f}$ is a $(2,-)$-matrix, then $A_{\hat{f}}=A_{f}$, i.e. $f$ is P-dual, and $f(r)=-\hat{f}\left(A_{f} r\right)+f(0)+\hat{f}(0), \forall r \in \mathbb{F}_{2}^{n}$.

Proof. Using Table 1, lemmas 2 and 4, if $f$ is selfdual or anti-selfdual,

$$
H^{\otimes n} i^{f(x+r)}=H^{\otimes n} i^{f(x)+2 A_{f} r \cdot x+f(r)-f(0)}=\alpha i^{f(x)+2 r \cdot x} .
$$

Similarly,

$$
H^{\otimes n} i^{f\left(x+A_{f} r\right)}=H^{\otimes n} i^{f(x)+2 A_{f}^{2} r \cdot x+f\left(A_{f} r\right)-f(0)}=\alpha i^{f(x)+2 A_{f} r \cdot x}, \quad \alpha \in \pm 1 .
$$

Then, as $H^{\otimes n}$ is self-inverse,

$$
\alpha i^{f(x)+2 r \cdot x-f(r)+f(0)}=\alpha^{-1} i^{f(x)+2 A_{f}^{2} r \cdot x+f\left(A_{f} r\right)-f(0)},
$$

from which $A_{f}^{2}=I$ and $f(r)=-f\left(A_{f} r\right)+2 f(0)$. For the converse, we have that

$$
H^{\otimes n} i^{f(x+r)}=H^{\otimes n} i^{f(x)+2 A_{f} r \cdot x+f(r)-f(0)}=i^{\hat{f}(x)+2 r \cdot x},
$$

and

$$
H^{\otimes n} i^{\hat{f}\left(x+A_{f} r\right)}=H^{\otimes n} i^{\hat{f}(x)+2 A_{\hat{f}} A_{f} r \cdot x+\hat{f}\left(A_{f} r\right)-\hat{f}(0)}=i^{f(x)+2 A_{f} r \cdot x} .
$$


Combining gives

$$
i^{\hat{f}(x)+2 r \cdot x-f(r)+f(0)}=i^{\hat{f}(x)+2 A_{\hat{f}} A_{f} r \cdot x+\hat{f}\left(A_{f} r\right)-\hat{f}(0)} .
$$

Let $\mathcal{E}_{f}$ be the eigenspace of $A_{f}$ associated with the eigenvalue 1 (we are working $\bmod 2$, so all eigenvectors have eigenvalue 1 ).

Lemma 5 If $f$ is selfdual or anti-selfdual, and $e \in \mathcal{E}_{f}$, then so is $f+2 e \cdot x$. Conversely, if both $f$ and $f+2 e \cdot x$ are selfdual or anti-selfdual, then $e \in \mathcal{E}_{f}$. Consequently, if $f$ is selfdual or anti-selfdual, then so are $\left|\mathcal{E}_{f}\right|$ binary linear offsets of $f$.

Proof. Assume $f(0)=0$. Then, from theorem 1, if $f$ is selfdual or anti-selfdual, then $f(r)+f\left(A_{f} r\right)=0$. For $e \in \mathcal{E}_{f}$ we have $A_{f} e=e$, so $\left(A_{f}+I\right) e=0, \bmod 2$, and $2\left(A_{f}+I\right) e=0, \bmod 4$. Therefore $f(r)+f\left(A_{f} r\right)+2\left(A_{f}+I\right) e \cdot r=0$. But $2\left(A_{f}+I\right) e \cdot r=2\left(A_{f}+I\right) r \cdot e$, so $(f(r)+2 r \cdot e)+\left(f\left(A_{f} r\right)+2 A_{f} r \cdot e\right)=0$, so $f+2 e \cdot x$ is selfdual. The converse is similarly proved, and the argument is easily generalised to $f(0)$ other than 0 .

Theorem 2 If $f$ is selfnegadual then $A_{f}$ is a $(3,3)$-matrix, and $f\left(A_{f} r\right)=w(r)+$ $f(0), \forall r \in \mathbb{F}_{2}^{n}$. Conversely, if $A_{f}$ is a $(3,3)$-matrix then $A_{\tilde{f}}=A_{f}, f$ is P-negadual, and $f(r)=\tilde{f}(r)-\tilde{f}\left(A_{f} r\right)+f(0)+w(r)$.

Proof. Using Table 1, lemmas 3 and 4 , if $f$ is selfnegadual,

$$
N^{\otimes n} i^{f(x)+2 A_{f} r \cdot x}=\alpha i^{f\left(x+A_{f} r\right)}=\alpha i^{f(x)+2 A_{f}^{2} r \cdot x+f\left(A_{f} r\right)-f(0)},
$$

and

$$
N^{\otimes n} i^{f(x+r)-f(r)+f(0)}=\alpha i^{f(x+r)+2 r \cdot x+w(r)-f(r)+f(0)}=\alpha i^{f(x)+2\left(A_{f}+I\right) r \cdot x+w(r)} .
$$

But, by lemma 4 , we can equate these equations. So, taking the righthand expressions of both lines we get $f(x)+2 A_{f}^{2} r \cdot x=f(x)+2\left(A_{f}+I\right) r \cdot x$ and $f\left(A_{f} r\right)-f(0)=w(r)$. Therefore $A_{f}^{2}=A_{f}+I$, mod 2, (implying that both $A_{f}$ and $A_{f}+I$ have order 3$)$, and $f\left(A_{f} r\right)=w(r)+f(0)$, and the first part of the theorem follows. For the second part we obtain, in a similar fashion,

$$
i^{\tilde{f}(x)+2 A_{\tilde{f}} A_{f} r \cdot x+\tilde{f}\left(A_{f} r\right)-\tilde{f}(0)}=i^{\tilde{f}(x)+2\left(A_{\tilde{f}}+I\right) r \cdot x+\tilde{f}(r)-f(r)+\tilde{f}(0)+f(0)+w(r)},
$$

leading to $A_{\tilde{f}} A_{f}=A_{\tilde{f}}+I$ and $f(r)=\tilde{f}(r)-\tilde{f}\left(A_{f} r\right)+f(0)+w(r)$. The argument is completed by observing that $A_{f}$ a $(3,3)$ matrix implies that $A_{f}+I$ has maximum rank, with inverse $A_{f}$. So, as $A_{\tilde{f}}\left(A_{f}+I\right)=I$, then $A_{\tilde{f}}=A_{f}$.

It is later shown in lemma 12 that, if $A_{f}$ is a $(3,3)$ matrix, then $n$ must be even.

From the equation $f\left(A_{f} r\right)=w(r)+f(0)$ of theorem 2, it is evident that, if $A_{f}$ is a $(3,3)$ matrix then $f$ is uniquely defined, to within a constant:

Corollary 1 If $f$ is selfnegadual then $f+2 e \cdot x$ is not selfnegadual $\forall e \in \mathbb{F}_{2}^{n}, e \neq 0$. 
It remains to derive an expression for the binary vector $b \in \mathbb{F}_{2}^{n}$ of (1). Let $\mu=\left(\mu_{0}, \mu_{1}, \ldots, \mu_{n-1}\right)$, where $\mu_{i}$ is the Hamming weight of column $i$ of $A_{f}$. Let $a \in \mathbb{F}_{2}^{n}$ be the diagonal of $A_{f}$, and let $\mathbf{1}$ be the all-ones vector of length- $n$.

Lemma 6 If $f$ is selfnegadual, then

$$
b=\frac{1}{2}\langle\mu+a+\mathbf{1}\rangle_{4},
$$

where $\langle *\rangle_{4}$ means reduce, $\bmod 4$.

Proof. Wlog we can restrict to $f(0)=0$. Then, from theorem 2, $f\left(A_{f} r\right)=w(r)$. Therefore, as $\left(A_{f}+I\right) A_{f}=I$, then $f(r)=w\left(\left(A_{f}+I\right) r\right)$. When $r$ has Hamming weight 1 , then $f(r)=(a+2 b) \cdot r$, and $w\left(\left(A_{f}+I\right) r\right)=\mu \cdot r-2 a \cdot r+1$. So $2 b=\mu+a+\mathbf{1}$.

Examples: Let $g=2 x_{0} x_{1}+x_{1}$, where $n=2$. Then $A_{g}$ is a $(3,3)$ matrix. Moreover $\mu=(1,2)$, and $a=(0,1)$. So $2 b=(1,2)+(0,1)+(1,1)=(2,0)$ and, from lemma $6, f=2 x_{0} x_{1}+2 x_{0}+x_{1}$ is selfnegadual.

Now let $g=2\left(x_{0} x_{1}+x_{0} x_{2}+x_{0} x_{5}+x_{1} x_{3}+x_{1} x_{5}+x_{2} x_{4}+x_{2} x_{5}+x_{3} x_{4}+x_{3} x_{5}+\right.$ $\left.x_{4} x_{5}\right)+x_{5}$, where $n=6$. Then $A_{g}$ is a $(3,3)$ matrix. Moreover $\mu=(3,3,3,3,3,6)$, and $a=(0,0,0,0,0,1)$. So $2 b=(4,4,4,4,4,8)=(0,0,0,0,0,0)$ and $f=g$ is selfnegadual.

Before continuing, we summarise function dualities of $f$ in terms of the associated orders of $A_{f}$. For instance, we indicate the property ' $A_{f}$ has an order' by the row 'ord $\left(A_{f}\right)$ ', and indicate the property ' $f$ is bent' by the column ' $f$ bent'. There is a $\sqrt{ }$ ('tick') symbol at the intersection of this row and column to indicate that one property implies the other. The - symbol indicates that the row/column properties do not imply each other. The $\sqrt{ }$ symbol is replaced by a number $(2$ or 3 ) if the order of $A_{f}$ or $A_{f}+I$ is known explicitly.

\begin{tabular}{l||l|l|l|l|l} 
property of: & $f$ & $f$ & $f$ & $f+\sum_{j} x_{j}$ & $f$ \\
\hline & bent & negabent & P-dual & P-dual & P-negadual \\
\hline $\operatorname{ord}\left(A_{f}\right)$ & $\sqrt{ }$ & - & 2 & - & 3 \\
$\operatorname{ord}\left(A_{f}+I\right)$ & - & $\sqrt{ }$ & - & 2 & 3
\end{tabular}

Remarks: The condition in the proof of theorem 2 that $A+I$ has maximum rank is important, as both $(3,-)$ and $(-, 3)$ matrices exist. For $A$ of order 3 , $\left(A^{3}+I\right)=\left(A^{2}+A+I\right)(A+I)$, and the condition that $(A+I)$ has maximum rank is equivalent to the condition that $A^{2}+A+I=0$.

Lemma 7 [1,13] An invertible binary symmetric matrix can be factored in the form $M M^{T}$ iff it has at least one nonzero term on the main diagonal.

If $f \in \operatorname{ZRM}(2, n)$ is such that $A_{f}$ has zero diagonal, then $f$ is a quadratic Boolean function.

Lemma 8 If $f$ is a bent quadratic Boolean function, then $A_{f}$ has even order.

Proof. If $A_{f}$ has odd order, $p$, then we can write $A_{f}=B^{2}$, where $B=A_{f}^{\frac{p+1}{2}}$. But, $B$ is symmetric as $A_{f}$ is symmetric so $A_{f}=B B^{T}$ which, by lemma 7 , is impossible as $A_{f}$ has no ones on its diagonal. 
Lemma 9 There are no selfnegadual quadratic Boolean functions.

Proof. By theorem 2, $A_{f}$ has order 3 if $f \in \operatorname{ZRM}(2, n)$ is selfnegadual. Therefore, by lemma $8, f$ cannot be a quadratic Boolean function.

Remark: The result of lemma 9 can also be deduced from the thesis of Van den Nest [17], in the context of $\mathbb{F}_{4}$-additive and $\mathbb{F}_{4}$-linear codes.

We identify an action which preserves the $(p, q)$ property of a matrix. An orthogonal matrix, $U$, satisfies $U U^{T}=I$. The set of $n \times n$ binary orthogonal matrices forms a group, $\mathcal{O}_{n}$, under multiplication. Then it is trivial to show that $U A U^{T}$ is a $(p, q)$-matrix iff $A$ is a $(p, q)$-matrix, and corollary 2 follows immediately from this observation.

Corollary 2 For $U \in \mathcal{O}_{n}$ and $f(x) \in Z R M(2, n)$, and by theorems 1 and 2, if $f(x)$ is selfdual then so is $f(U x)$ and, if $f(x)$ is selfnegadual then so is $f(U x)$.

\section{Graphs and code dualities}

Let $A$ be an $n \times n$ symmetric binary matrix. Then $A$ can be used to generate codes, and can also be interpreted as an adjacency matrix for an undirected graph, where the graph of $A$ has loops if its diagonal is non-zero. The next lemma is well-known.

Lemma 10 If $A$ has zero diagonal, and if $A^{2}=I$ then the linear space generated by the rows of $A+I$ is a self-orthogonal binary linear code of dimension rank $(A+I)$. In particular, if $A$ is the adjacency matrix for a bipartite graph with equal-size partitions, then $A+I$ generates a selfdual binary linear code.

Proof. The rows of $A$ are pairwise orthogonal as $A^{2}=I$. Therefore the rows of $A+I$ are pairwise orthogonal as $A$ is symmetric. The linear space generated by $A+I$ is self-orthogonal because $(A+I)(A+I)^{T}=0$. For the last part, $A=\left(\begin{array}{cc}0 & P \\ P^{T} & 0\end{array}\right)$, $P$ square, so $A+I$ spans a self-dual code.

An additive code over $\mathbb{F}_{4}=\{0,1, \omega, \bar{\omega}=\omega+1\}$ of length $n$ is a $\mathbb{F}_{2}$-linear subgroup of $\mathbb{F}_{4}^{n}$. We define the dual of the code $\mathcal{C}$ with respect to the trace inner product as $\mathcal{C}^{\perp}=\left\{\boldsymbol{u} \in \mathbb{F}_{4}^{n} \mid \sum_{i=1}^{n}\left(u_{i} \overline{c_{i}}+\overline{u_{i}} c_{i}\right)=0\right.$ for all $\left.\boldsymbol{c} \in \mathcal{C}\right\}$, and say that it is selfdual if $\mathcal{C}=\mathcal{C}^{\perp}$. A linear code over $\mathbb{F}_{4}$ which is selfdual with respect to the Hermitian inner product, i.e., $\boldsymbol{u} \cdot \overline{\boldsymbol{v}}$, is also selfdual additive with respect to the trace inner product. However, most selfdual additive codes are not $\mathbb{F}_{4}$-linear. Two selfdual additive codes over $\mathbb{F}_{4}$ are equivalent if we can obtain one from the other by permuting, scaling, and conjugating coordinates. Equivalence of selfdual linear codes is defined similarly, with the exception that conjugating single coordinates is not permitted.

It is known [17] that any selfdual additive code has a standard form $n \times n$ generator matrix of the form $A+\omega I$, where $I$ is the identity matrix and $A$ is a binary symmetric matrix. It is also known [17] that two codes are equivalent iff the corresponding graphs are related via local complementation (LC) [2]. Given a generator matrix of standard form, conjugating a coordinate is equivalent to complementing a diagonal element in $A$. Hence, for selfdual additive codes considered up to equivalence, this diagonal can always be set to zero. For selfdual linear 
codes (represented by overdefined $n \times n$ generator matrices), the diagonal is of importance and can in fact never be zero.

Lemma 11 [17] For $A$ and $B$ symmetric, a Hermitian selfdual $\mathbb{F}_{4}$-additive code, generated by $A+\omega B$, is $\mathbb{F}_{4}$-linear iff $A A^{T}+B B^{T}+A B^{T}=0$. Moreover, if $B=I$, this condition reduces to $A^{2}+A+I=0$.

Corollary $3 A+\omega I$ is the codespace of a Hermitian selfdual $\mathbb{F}_{4}$-linear code iff $A$ is a $(3,3)$ matrix.

Proof. Follows from lemma 11 as the condition $A^{2}+A+I=0$ immediately implies that both $A$ and $A+I$ have order 3 .

Let $G_{A}$ be the $n$-vertex graph of $A$ with an edge between $i$ and $j$ iff $a_{i j}=1$, and a loop at vertex $j$ iff $a_{j j}=1$.

Lemma 12 If $A$ is a $(3,3)$ matrix, then all vertices of $G_{A}$ have odd degree, disregarding loops. In such a case, $n$, the number of vertices, must be even.

Proof. Since $\mathrm{A}$ is a $(3,3)$ function, $A^{2}=A+I$. Hence the diagonal entries in $A^{2}$ must be the complements of the diagonal entries of $A$. This criterion is satisfied iff there is an odd number of 1 s among the off-diagonal entries in each row of $A$. The number of edges of a graph equals the sum of the vertex degrees divided by 2 . But, if all vertex degrees are odd, then $n$ must be even.

Remark: It is also a well-known fact that all codewords of selfdual $\mathbb{F}_{4}$-linear codes have even weight, which amounts to the same thing.

It is known [7] that local complementation (LC) acting on $G_{A}$, where loops are ignored, preserves, to within equivalence, the $\mathbb{F}_{4}$ additive selfdual code generated by $A+\omega I$. However, for those additive codes that are also linear codes, one has to modify local complementation to take account of loops, so that the additive code remains linear after local complementation. We first describe this modified form of local complementation $\left(\mathrm{LC}^{*}\right)$ and then prove that it preserves linearity of the $\mathbb{F}_{4}$ codespace. Let $\mathcal{N}_{j} \subset\{0,1, \ldots, n-1\}$ be the set of vertices that are neighbours to vertex $j$ in $G_{A}$ (not including $j$ itself). Let $L^{*}$ at vertex $j$ of $G_{A}$ produce the graph $G_{A^{\prime}}$.

\section{Modified local complementation ( $\left.\mathrm{LC}^{*}\right)$ :}

$\mathrm{LC}^{*}$ on $G_{A}$ at vertex $j$ is realised by

$$
\begin{array}{lc}
a_{i k}^{\prime}=a_{k i}^{\prime}=a_{i k}+1 & i, k \in \mathcal{N}_{j}, \\
a_{i k}^{\prime}=a_{k i}^{\prime}=a_{i k} & \text { otherwise. } \\
a_{i i}^{\prime}=a_{i i}+1 & i \in j \cup \mathcal{N}_{j}, \\
a_{i i}^{\prime}=a_{i i} & \text { otherwise. }
\end{array}
$$

Lemma 13 Let $G_{A^{\prime}}$ be the graph resulting from $L C^{*}$ on $G_{A}$ at some vertex. If $A$ is a $(3,3)$-matrix, then $A^{\prime}$ is also a $(3,3)$-matrix.

Proof. Let $\mathrm{LC}^{*}$ act on $G_{A}$ at vertex $j$. Let $D=\left(d_{i k}\right)$ and $V=\left(v_{i k}\right)$ be $n \times n$ binary matrices such that $d_{j j}=1$ and $d_{i k}=0$ otherwise, and $v_{i k}=1$, $\forall i, k \in \mathcal{N}_{j}, i \neq k$, and $v_{i k}=0$ otherwise. Then, by the $\mathrm{LC}^{*}$ rule, one can see that $A^{\prime}=A+D+V$. $A^{\prime}$ is a $(3,3)$-matrix iff $A^{\prime 2}=A^{\prime}+I$. But $A^{\prime 2}=(A+D+V)^{2}=$ $\left(A^{2}+D^{2}+V^{2}\right)+(A D+D A)+(A V+V A)+(D V+V D)$. But $A^{2}=A+I$ 
Table 2 Number of selfdual linear codes over $\mathbb{F}_{4}$ of length $n[5,9,10,14]$

\begin{tabular}{ccccccccccc}
\hline$n$ & 2 & 4 & 6 & 8 & 10 & 12 & 14 & 16 & 18 & 20 \\
\hline & 1 & 1 & 2 & 3 & 5 & 10 & 21 & 55 & 245 & 3427 \\
\hline
\end{tabular}

as $A$ is a $(3,3)$-matrix. Moreover it is easily verified that $D^{2}=D$. Using lemma $12, V^{2}=V$, and $A D+D A=A V+V A . D$ and $V$ are row/column disjoint, so $D V+V D=0$. Therefore, $A^{\prime 2}=A+I+D+V=A^{\prime}+I$, as required.

The modified local complementation proposed in [3] differs crucially from ours - when doing a local complementation at vertex $j$, they do not flip the diagonal at $j$, whereas we do.

\section{Code classification}

Selfdual linear codes over $\mathbb{F}_{4}$ have been classified up to length $16[5,14]$. As a consequence of lemma 11 and corollary 3 , we can use the correspondence to $(3,3)$ matrices to devise a new algorithm and classify codes of length 18 . During the process of extending the result to length 20 , we became aware that codes of length 18 and 20 have already been classified independently in two preprints recently made available online $[9,10]$. However, we still give an overview of our approach, since it gives different theoretical insights and highlights the connection between selfdual codes and selfnegadual Boolean functions.

It has been shown in theorem 3.1 of [11] that all $(2,-)$ symmetric matrices are orthogonally equivalent to one of a small set of canonical forms. We use a similar proof method to obtain the following theorem.

\section{Theorem $3^{1}$}

Let $A$ be a $(3,3)$ matrix of size $n \times n$. Then $A$ is orthogonally congruent to the canonical form $C_{n}=\left(\begin{array}{ll}0 & 1 \\ 1 & 1\end{array}\right) \otimes I_{n / 2}$, where $I_{n / 2}$ is the $n / 2 \times n / 2$ identity matrix, i.e. there always exists an orthogonal matrix, $U \in \mathcal{O}_{n}$, such that

$$
A=U\left(\left(\begin{array}{ll}
0 & 1 \\
1 & 1
\end{array}\right) \otimes I_{n / 2}\right) U^{T} \text {. }
$$

Proof. We make repeated use of the identity $A^{2}+A+I=0$. Let $v=$ $(1,0,0, \ldots, 0)$ be of length $n$. Then $v v^{T}=1$. We aim to convert, in $t$ steps, $A$ to $C_{n}$ by successive actions of the form $V_{t} A V_{t}^{T}$, where $V_{t} \in \mathcal{O}_{n}$. Then $U=V_{t-1} \ldots V_{1} V_{0}$. Moreover, wlog we consider only the case of $A=\left(a_{i j}\right)$ where $a_{00}=0$ because, when $a_{00}=1$ then we can orthogonally transform $A^{2}=A+I$ instead to obtain $C_{n}$. Then $C_{n}=U A^{2} U^{T}=U(A+I) U^{T}=U A U^{T}+I$, and so $(X U) A\left(U^{T} X\right)=C_{n}$.

Given the above we have that $v A v^{T}=0$ and $v A^{2} v^{T}=1$. We create the $n \times n$ matrix,

$$
V=\left(\begin{array}{c}
v \\
v A=a_{0} \\
v_{2} \\
v_{3} \\
\cdots \\
v_{n-1}
\end{array}\right)
$$

\footnotetext{
1 We thank Prof. Alexander Pott for suggesting the proof strategy used for this theorem.
} 
and we wish to choose $v_{j}$ so that $v v_{j}^{T}=v A v_{j}^{T}=v_{k} v_{j}^{T}=0,2 \leq j, k<n, j \neq k$, in which case $V \in \mathcal{O}_{n}$. If we can do this then

$$
V^{T} A V=\left(\begin{array}{ll}
0 & 1 \\
1 & 1
\end{array}\right) \oplus B
$$

where $B$ is a $(3,3)$ matrix of size $(n-2) \times(n-2)$. The proof follows by induction on $B$, to obtain $C_{n}$.

It remains to show that we can always select the $v_{j}$ so that $V \in \mathcal{O}_{n}$. Let us further assume that $a_{01}=0$. If this is not the case then it can always be made so by the action $P A P^{T}$, where $P$ is a row/column permutation satisfying $P P^{T}=I$, iff $a_{0} \neq(011 \ldots 11)$. Let us assume, for now, that $a_{0} \neq(011 \ldots 11)$. Then, by orthogonality, we require $V$ to be of the form $V=1 \oplus R$, where $R$ is an $(n-1) \times(n-1)$ orthogonal matrix. But non-trivial binary orthogonal matrices are of even dimension so $R$ is orthogonal iff $R=Q\left(I_{k} \oplus R^{\prime}\right) Q^{T}$, where $I_{k}$ is the $k \times k$ identity, $k$ odd, $Q$ is some permutation, and $R^{\prime}$ is an $(n-1-k) \times(n-1-k)$ orthogonal matrix. For suitable $k$ we can make $R^{\prime}$ orthogonal by choosing it to be $R^{\prime}=J_{n-1-k}+I_{n-1-k}$, were $J_{k}$ is the all-ones matrix of dimension $k$, on condition that $a_{0}=\left(0,0, a_{02}, \ldots, a_{0(n-1)}\right)$ has odd weight, which it does by lemma 12 . Permutation $Q$ always exists, so $R$ can be made orthogonal, and therefore $V$ can be made orthogonal. We just need to show that $a_{0} \neq(011 \ldots 11)$ can be avoided. Imagine that $A$ is such that $a_{0}=(011 \ldots 11)$. Then swap row and column 0 of $A$ with row and column $j>0$, and call this $D$. This is an orthogonal conjugation so $D$ is also $(3,3)$. Then $D=\left(d_{i j}\right)$ has $d_{00}=1$ so now orthogonally conjugate $D^{2}$, as mentioned previously. Self-duality of the associated $\mathbb{F}_{4}$-additive code [7] implies that $w\left(d_{0}+d_{j}\right)>1$, so the first row of $D^{2}$ is never equal to $(011 \ldots 11)$.

Example (to illustrate proof of theorem 3) Let $A=\left(\begin{array}{l}111111 \\ 100101 \\ 100011 \\ 110010 \\ 101100 \\ 111000\end{array}\right)$. Then $A$ is a $(3,3)$ matrix. As $a_{00}=1$ we conjugate $A^{2}=\left(\begin{array}{l}011111 \\ 110101 \\ 101011 \\ 110110 \\ 101110 \\ 111001\end{array}\right)$ instead. As $a_{0}=(011111)$ we swap $a_{0}$ and, say, $a_{2}$ to get $D$. As $d_{00}=1$ we orthogonally conjugate $D^{2}=$ $\left(\begin{array}{l}001011 \\ 001101 \\ 11111 \\ 0111010 \\ 101100 \\ 111000\end{array}\right)$. Choose $V=\left(\begin{array}{l}100000 \\ 001011 \\ 010000 \\ 000111 \\ 001101 \\ 00110\end{array}\right)$. Then $V D V^{T}=\left(\begin{array}{c}01 \\ 11\end{array}\right) \oplus\left(\begin{array}{l}0010 \\ 0001 \\ 1010 \\ 0101\end{array}\right) \ldots$ and so on

Corollary 4 (of theorem 3) Given two $(3,3)$ matrices, $A$ and $A^{\prime}$, of size $n \times n$, there always exists an orthogonal matrix $U \in \mathcal{O}_{n}$ such that $A^{\prime}=U A U^{T}$.

By our classification, we verify theorem 3 numerically for $n \leq 18$. Also note that it has been shown by Janusz [12] that all selfdual binary codes of length $n$ are equivalent under the action of $\mathcal{O}_{n}$. It is known [12] that $\mathcal{O}_{n}$ is generated by all matrices of the form $P M$, where $P$ is a permutation matrix and $M=$ $\left(\begin{array}{cc}I_{n-4} & 0 \\ 0 & I_{4}+J_{4}\end{array}\right)$, where $J_{4}$ is the $4 \times 4$ all-one matrix. As a canonical representative for selfdual $\mathbb{F}_{4}$-linear codes of length $n$, we choose the matrix $C_{n}=\left(\begin{array}{ll}0 & 1 \\ 1 & 1\end{array}\right) \otimes I_{n / 2}$. Starting from $C_{n}$, we then apply orthogonal transforms until one representative from each equivalence class has been found. (Which is verified by checking the mass 
formula [14].) In practice, we achieve this by generating $C^{\prime}=(P M) C_{n}(P M)^{T}$ for all the $\left(\begin{array}{c}n / 2 \\ 4\end{array}\right)$ non-trivial permutations $P$. Then $C^{\prime}$ is treated as a graph with loops and checked for isomorphism against all previously seen graphs. If $C^{\prime}$ is new, the corresponding code is output, and the complete $\mathrm{LC}^{*}$-orbit of $C^{\prime}$, using modified local complementation, is generated and stored. (This is not strictly necessary, but speeds up the algorithm, since $\mathrm{LC}^{*}$-operations are faster than orthogonal transforms.) We proceed recursively, generating matrices $(P M) C^{\prime}(P M)^{T}$, and so on, until all codes are found. With this algorithm, classifying all codes of length $n \leq 18$ was achieved in about two hours of CPU time on a standard desktop computer.

\section{Discussion - further work}

It is of interest to consider further how the order of a symmetric binary matrix relates to its associated code and/or graph. We offer here one brief observation that may be worth pursuing:

The optimal Hermitian selfdual additive code over $\mathbb{F}_{4}$ for $n=6$ is the hexacode, with distance 4 . It can be generated by the matrix $A_{f}+\omega I$, with associated function, $f \in \operatorname{ZRM}(2,6)$ given by $f=2\left(x_{0} x_{1}+x_{0} x_{2}+x_{1} x_{2}+x_{3} x_{4}+x_{3} x_{5}+\right.$ $\left.x_{4} x_{5}+x_{0} x_{3}+x_{1} x_{4}+x_{2} x_{5}\right)$, where we zero the diagonal of $A_{f}$. The graph, $G_{A_{f}}$, is in so-called 'nested-clique' form, specifically the '2-clique of 3-cliques' graph [6]. Let $A_{g}=\left(A_{f}+I\right)^{2}$. Then $g=x_{0} x_{1}+x_{0} x_{2}+x_{1} x_{2}+x_{3} x_{4}+x_{3} x_{5}+x_{4} x_{5}$, and $G_{A_{g}}$ is the disjoint sum of two 3-cycles, $C_{3} \oplus C_{3}$. Similarly, the dodecacode is the optimal code for $n=12$ with distance 6 . It can be represented by a graph, $G_{A_{f}}$, which is a '3-clique of 4-cliques' [6]. Let $A_{g}=\left(A_{f}+I\right)^{2}$. Then $G_{A_{g}}$ is the disjoint sum of two 6-cycles, $C_{6} \oplus C_{6}$. A near-optimal code for $n=18$, and with distance 6 , can be represented by a graph, $G_{A_{f}}$, which is a '2-clique of 3-cliques of 3-cliques' [6] (the optimal code has distance 8). Let $A_{g}=\left(A_{f}+I\right)^{6}$. Then $G_{A_{g}}$ is the disjoint sum of two 9-cycles, $C_{9} \oplus C_{9}$. Moreover, for $A_{g^{\prime}}=\left(A_{f}+I\right)^{2}$, then $G_{A_{g^{\prime}}}$ is the disjoint sum of two ' 3 -clique of 3-cliques', which is an optimal code for $n=9$ with distance 4 . These preliminary observations motivate a classification of optimal (or near-optimal) codes and of nested-clique graphs that arise as 'roots' of the disjoint cycles.

Acknowledgements: The authors would like to thank the reviewers for their helpful suggestions which improved the quality of the submission. We would also like to thank Prof. Alexander Pott for helping with one of the proofs.

\section{References}

1. Albert, A.A.: Symmetric and alternating matrices in an arbitrary field. AMS Trans. 43 (1938) 386-436

2. Bouchet, A.: Graphic presentations of isotropic systems. J. Combin. Theory Ser. B 45(1) (1988) 58-76

3. Brijder, R., Hoogeboom J.H.: Pivot and Loop Complementation on Graphs and Set Systems. Theory and applications of models of computation, Lecture Notes in Computer Science, LNCS 6108 (2010) 151-162

4. Carlet, C., Danielsen, L.E., Parker, M.G., Solé, P.: Self-dual bent functions. Int. J. Inform. and Coding Theory 1(4) (2010) 384-399 
5. Conway, J.H., Pless, V., Sloane, N.J.A.: Self-dual codes over GF(3) and GF(4) of length not exceeding 16. IEEE Trans. Inform. Theory 25(3) (1979) 312-322

6. Danielsen, L.E., Parker, M.G.: Spectral orbits and peak-to-average power ratio of Boolean functions with respect to the $\{I, H, N\}^{n}$ transform. Sequences and Their Applications SETA 2004, Lecture Notes in Computer Science, LNCS 3486, (2005) 373-388

7. Danielsen, L.E., Parker, M.G.: On the classification of all self-dual additive codes over GF(4) of length up to 12. Journal of Combinatorial Theory, Series A, 113(7) Oct. (2006) 1351-1367

8. Danielsen, L.E., Parker, M.G., Solé, P.: The Rayleigh quotient of bent functions. 12th IMA International Conference on Cryptography and Coding, 15-17 Dec. 2009, Cirencester, UK, Lecture Notes in Computer Science, LNCS 5921 (2009) 418-432

9. Harada, M., Lam, C., Munemasa, A., Tonchev, V.D.: Classification of generalized Hadamard matrices $\mathrm{H}(6,3)$ and quaternary Hermitian self-dual codes of length 18. Electronic J. Combinatorics 17 (2010), \#R171.

10. Harada, M., Munemasa, A.: Classification of quaternary Hermitian self-dual codes of length 20. IEEE Trans. Inform. Theory 57(6) (2011) 3758-3762

11. Hou, X-D.: Classification of self dual quadratic bent functions. Designs, Codes and Cryptography, 63(2), (2012), 183-198

12. Janusz, G.J.: Parametrization of self-dual codes by orthogonal matrices. Finite Fields Appl. 13(3) (2007) 450-491

13. MacWilliams, J.: Orthogonal Matrices Over Finite Fields. The American Mathematical Monthly. 76(2) (1969) 152-164

14. MacWilliams, F.J., Odlyzko, A.M., Sloane, N.J.A., Ward, H.N.: Self-dual codes over GF(4). J. Combin. Theory Ser. A 25(3) (1978) 288-318

15. Parker, M.G., Pott, A.: On Boolean functions which are bent and negabent. S.W. Golomb, G. Gong, T. Helleseth and H.Y. Song, (Eds.), Sequences, Subsequences, and Consequences, International Workshop, SSC 2007, Los Angeles, CA, USA, May 31 - June 2, 2007, Lecture Notes in Computer Science, LNCS 4893 (2007)

16. Schmidt, K-U., Parker, M.G., Pott, A.: Negabent Functions in the Maiorana-McFarland Class. Sequences and Their Applications - SETA 2008, University of Kentucky, Lexington, KY, Lecture Notes in Computer Science, LNCS 5203 Sept. (2008) 14-18

17. Van den Nest, M.: Local Equivalence of Stabilizer States and Codes. PhD thesis, K. U. Leuven, Belgium (May 2005) 